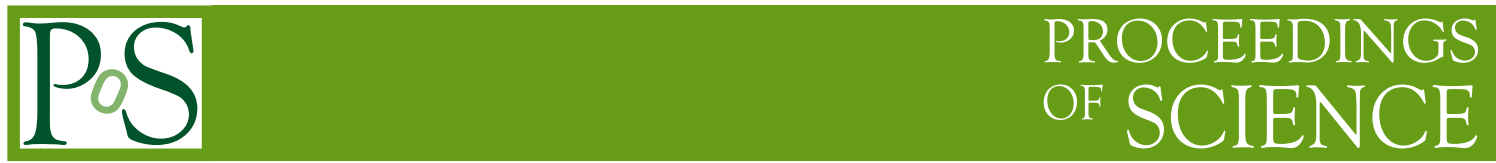

\title{
Other searches for a high mass Higgs boson at Tevatron
}

\author{
Antonio Limosani*i \\ University of Melbourne \\ E-mail: antoniol@unimelb.edu.au
}

\begin{abstract}
We report on the search at the Tevatron for Higgs bosons with masses roughly $135 \mathrm{GeV}$ and above in final states other than in the $W$-boson pair decay channel where the $W$-bosons decay to leptons. These include: Semileptonic decay channel $\left(H \rightarrow W W \rightarrow l v q q^{\prime}\right)$; Hadronic tauon decay channels $\left(H \rightarrow W W \rightarrow l v \tau_{h} v\right)$; Tri-lepton final states $(W H \rightarrow W W W \rightarrow l v l v l v$ and $Z H \rightarrow$ $\left.Z W W \rightarrow l l l v q q^{\prime}\right)$; and Four-lepton final states $(H \rightarrow Z Z \rightarrow 4 l$ and $Z H \rightarrow Z W W \rightarrow l l l v l v)$. The searches utilise $5.4-8.2 \mathrm{fb}^{-1}$ of data.
\end{abstract}

The 2011 Europhysics Conference on High Energy Physics-HEP 2011,

July 21-27, 2011

Grenoble, Rhône-Alpes France

\footnotetext{
${ }^{*}$ Speaker.

${ }^{\dagger}$ On behalf of the CDF and D0 collaborations
} 


\section{Introduction}

The vector gauge bosons mediating the weak force, the $W$ and $Z$, are massive. Within the Standard Model of Particle Physics (SM) their masses arise through the combination of electroweak spontaneous symmetry breaking (SSB) and the Higgs mechanism. The latter posits the presence of a scalar field and an associated particle, the as yet to be observed Higgs boson. The Higgs mechanism is also able to provide mass to the fundamental fermions through Yukawa couplings. The discovery of the Higgs boson would unambiguously confirm electroweak SSB within the SM. The mass of the Higgs boson is a free parameter in the SM. Indirect limits based on precision electroweak data constrain it to be less than about $185 \mathrm{GeV}$ while a direct search by LEP excluded it below $114 \mathrm{GeV}$. At the Tevatron, production of the Higgs boson can proceed either via gluongluon fusion $(g g H)$, massive vector boson fusion (VBF) or associated production $(W H$ or $Z H)$.

The distinction between a low and high mass Higgs is made at the point where the Higgs decay branching fraction to $W$-boson pairs and that to $b$-quark pairs are equal, which is roughly at $135 \mathrm{GeV}$. In the high mass region the most sensitive channel is the Higgs decay to $W$-boson pairs, where the bosons decay leptonically. This is due to the favourable final state topology of two charged leptons and missing transverse energy that yields good control of the background without sacrificing too much signal. In this paper we summarise Tevatron results that fall into the next tier of sensitivity where either backgrounds are much larger or where signal is diminished. These channels include: Semileptonic decay $\left(H \rightarrow W W \rightarrow l v q q^{\prime}\right)$; Hadronic tauon decay channels $(H \rightarrow$ $\left.W W \rightarrow l v \tau_{h} v\right)$; Tri-lepton final states $\left(W H \rightarrow W W W \rightarrow l v l v l v\right.$ and $\left.Z H \rightarrow Z W W \rightarrow l l l v q q^{\prime}\right)$; and Four-lepton final states $(H \rightarrow Z Z \rightarrow 4 l$ and $Z H \rightarrow Z W W \rightarrow l l l v l v)$. The addition of these channels improves the overall sensitivity of the combined Higgs search at the Tevatron.

\section{Analysis}

The Semileptonic decay channel is explored by the D0 collaboration using $5.4 \mathrm{fb}^{-1}$ of data, through reconstruction of a high $p_{T}$ charged lepton, at least two jets and some missing transverse energy. Pre-selection criteria provide a signal to background ratio $(S: B)$ of 1 to 1000 , with the vector boson plus jets background process dominant. A random forest of decision trees based on several kinematic quantities is used to improve the ratio to 1 to 100 [1].

In the Hadronic decay channel, D0, in $7.3 \mathrm{fb}^{-1}$ of data, use a Neural Network (NN) output to identify tau hadronic decays. The NN is optimised separately for 1- and 3-prong decay modes of the tau, which typically yields an identification efficiency of $55 \%$ and a $2 \%$ fake rate. The analysis allows for up to one jet in the final state. Another NN is used to separate the Higgs signal from the dominant $W$-boson plus jets background. The $S: B$ is about 1 to 500 for a Higgs mass of $165 \mathrm{GeV}$ [2]. CDF similarly perform a search in this channel using $8.2 \mathrm{fb}^{-1}$ of data without a requirement on the number of jets. They employ a Boosted Decision Tree trained on kinematic and Tau identification variables. The analysis is performed separately for electron and muon channels. The $S: B$ is about 1 to 500 [3].

Searches in the tri-lepton final state channel are performed by CDF using $8.2 \mathrm{fb}^{-1}$. To improve the sensitivity the analysis is separated based on the reconstructed mass of the same flavor opposite sign leptons, where events that are within (SFOS-Z) or outside (non-SFOS-Z) a $10 \mathrm{GeV}$ window 
around the $Z$ mass are distinguished. Events with a reconstructed $Z$ are further divided into two categories based on the number of jets in the event: only one jet (SFOS-Z-1) and two or more jets (SFOS-Z-2). SFOS-Z-1, SFOS-Z-2 and non-SFOS-Z yield $S: B$ of 1:100, 1:30 and 1:17 respectively for a Higgs mass of $165 \mathrm{GeV}$. For non-SFOS-Z the dominant background is $W Z$ production. The channels are relatively clean but suffer from low expected signal yields, typically of the order of a single event in this dataset [4].

Searches in the four-lepton final state channel are performed by CDF using $8.2 \mathrm{fb}^{-1}$. The dominant contribution is from $g g \rightarrow H \rightarrow Z Z \rightarrow 4 l$. Three final state topologies are reconstructed: $4 e, 4 \mu$ and $2 e 2 \mu$. At Higgs masses below twice that of the $Z$, one or both of the $Z$ must be non-resonant, as such to gain sensitivity essentially no requirements are imposed on the dilepton invariant mass. The lepton that triggers the event is required to have a $p_{T}$ of $20 \mathrm{GeV}$. Lower thresholds requirements on the $p_{T}$ of the leptons at $10 \mathrm{GeV}$ limit dilepton masses to greater than 20 $\mathrm{GeV}$, while they are bounded from above at $140 \mathrm{GeV}$. A reasonable acceptance to $Z H \rightarrow Z W W$ is also gained. The search is conducted in the invariant mass of the four leptons, where the dominant background is from the non-resonant $Z Z$ in the Standard Model. The four lepton invariant mass is plotted in Fig 1, where over the whole mass range for a Higgs mass of 150 and $190 \mathrm{GeV}$ the $\mathrm{S}: \mathrm{B}$ is 1:17 and 1:25 respectively [5].

For all channels no excess above background is observed. Expected and observed limits are plotted in Figures 1b-e for all the channels presented.

\section{Conclusion}

In all channels data yields were consistent with those expected from SM background processes. The channels improve the overall Tevatron sensitivity to the discovery of the Higgs boson.

\section{References}

[1] V. M. Abazov et al. [The D0 Collaboration], Phys. Rev. Lett. 106 (2011) 171802

[2] V. M. Abazov et al. [The D0 Collaboration], D0 Note 6179-CONF

[3] T. Aaltonen et al., [CDF Collaboration], CDF Public Note 10597

[4] T. Aaltonen et al., [CDF Collaboration], CDF Public Note 10599

[5] T. Aaltonen et al., [CDF Collaboration], CDF Public Note 10573 


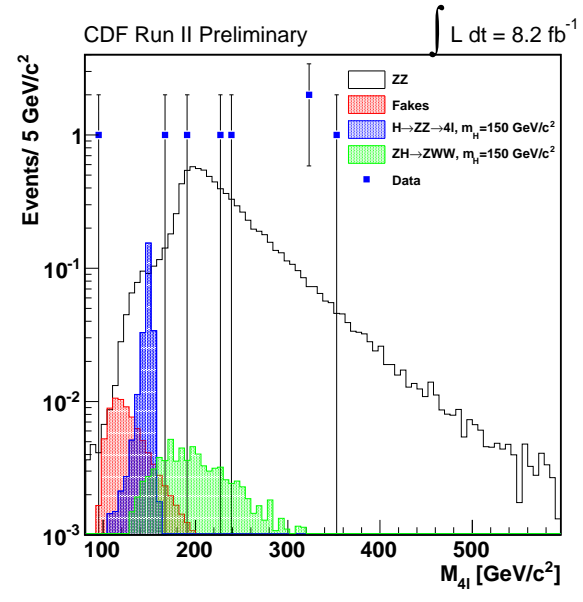

(a)

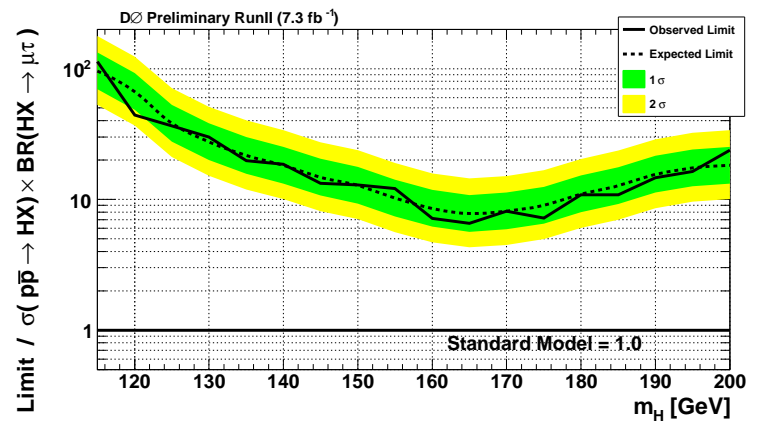

(c)

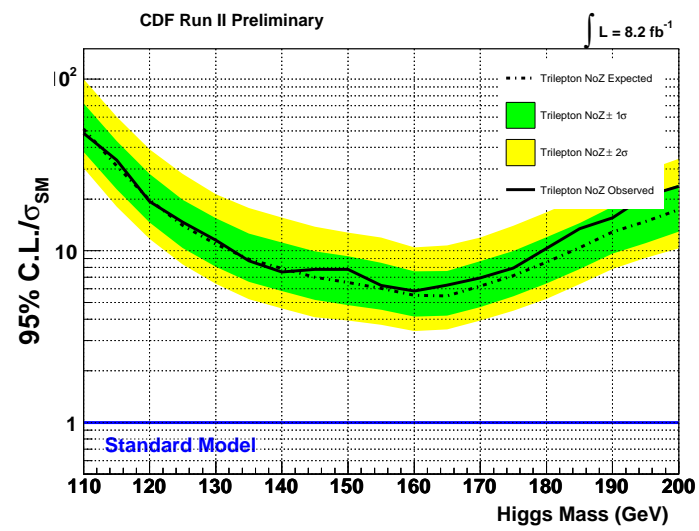

(e)

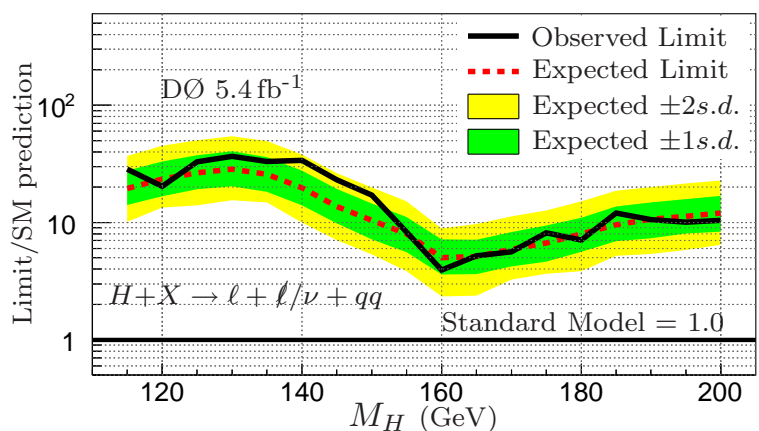

(b)

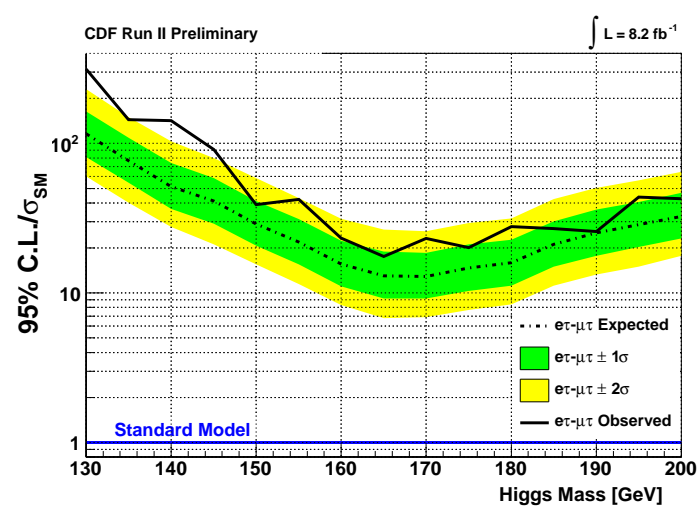

(d)

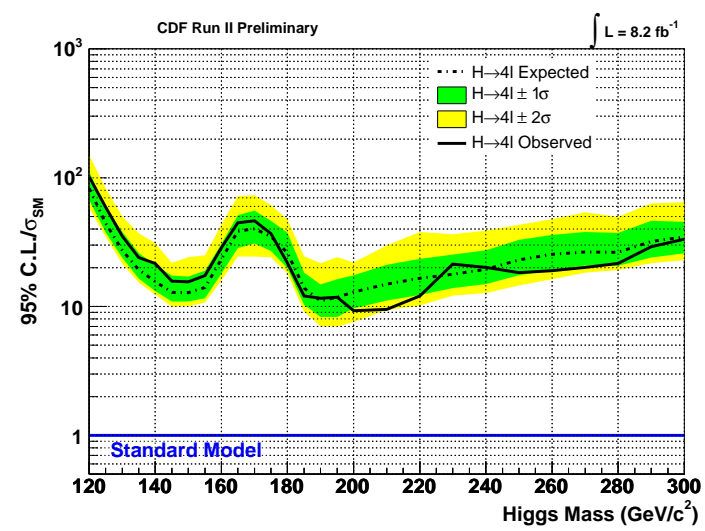

(f)

Figure 1: (a) The distribution of the four-lepton invariant mass as measured in data. Overlayed are estimated contributions from non-resonant $Z Z$ production, fakes component, and from the $H \rightarrow Z Z$ and $Z H \rightarrow Z W W$ four-lepton final state for a Higgs mass of $150 \mathrm{GeV} / c^{2}$. The expected and observed limits of the Higgs production cross section normalised to the SM prediction for Higgs masses for: (b) D0 $H \rightarrow W W \rightarrow l v q q^{\prime}$; (c) D0 $H \rightarrow W W \rightarrow l v \tau_{h} v$; (d) CDF $H \rightarrow W W \rightarrow l v \tau_{h} v$; (e) CDF $W H \rightarrow W W W \rightarrow l v l v l v$ and $Z H \rightarrow$ $Z W W \rightarrow l l l v q q^{\prime}$; and (f) $\mathrm{CDF} H \rightarrow Z Z \rightarrow 4 l$ and $Z H \rightarrow Z W W \rightarrow l l l v l v$. 